\title{
The origin of single low-mass WDs: another problem that consequential angular momentum loss in CVs might solve
}

\author{
M. Zorotovic, ${ }^{1 \star}$, M.R. Schreiber ${ }^{1}$ \\ ${ }^{1}$ Instituto de Física y Astronomía, Universidad de Valparaíso, Av. Gran Bretaña 1111, Valparaíso, Chile
}

Accepted 2016 November 21. Received 2016 November 18; in original form 2016 September 30

\begin{abstract}
Low-mass helium-core white-dwarfs (WDs) with masses below $0.5 \mathrm{M}_{\odot}$ are known to be formed in binary star systems but unexpectedly a significant fraction of them seem to be single. On the other hand, in Cataclysmic Variables (CVs) a large number of low-mass WD primary stars is predicted but not observed. We recently showed that the latter problem can be solved if consequential angular momentum loss causes especially CVs with low-mass WDs to merge and form single stars. Here we simulate the population of single WDs resulting from single star evolution and from binary star mergers taking into account these new merging CVs. We show that according to the revised model of $\mathrm{CV}$ evolution, merging CVs might be the dominant channel leading to the formation of low-mass single WDs and that the predicted relative numbers are consistent with observations. This can be interpreted as further evidence for the revised model of CV evolution we recently suggested. This model includes consequential angular momentum loss that increases with decreasing WD mass and might not only explain the absence of low-mass WD primaries in CVs but also the existence of single low-mass WDs.
\end{abstract}

Key words: white dwarfs - binaries: close - novae, cataclysmic variables -

\section{INTRODUCTION}

The main sequence (MS) lifetime of the progenitors of helium-core white dwarfs (WDs) with masses below $0.5 \mathrm{M}_{\odot}$, hereafter low-mass WDs, largely exceeds the Hubble time. However, approximately 10 per cent of all WDs in the solar neighbourhood are low-mass WDs (e.g. Kepler et al. 2007). The dominant evolutionary channel producing these lowmass WDs is common envelope (CE) evolution of close binary stars, which can cause the ejection of the envelope of a giant star much earlier than it is possible in standard single star evolution. If the WD progenitor fills its Roche-lobe on the first giant branch, dynamically unstable mass transfer leads to the formation of a gaseous envelope around the helium core of the giant and the companion star, which is expelled at the expense of orbital energy and angular momentum within $<_{\sim} 10^{3}$ yrs (e.g. Webbink 2008).

Indeed, observations show that low-mass WDs are typically members of close binary stars with either a MS star companion (Rebassa-Mansergas et al. 2011) or a second WD in a close orbit (Marsh, Dhillon \& Duck 1995). However, while the binary fraction is 100 per cent for extremely lowmass WDs $\left(\mathrm{M}_{\mathrm{WD}}<0.25 \mathrm{M}_{\odot}\right.$, e.g. Brown et al. 2010), there exists a population of single low-mass WDs with masses

^ E-mail: mzorotovic@dfa.uv.cl (MZ)
$0.25 \mathrm{M}_{\odot}<\mathrm{M}_{\mathrm{WD}}<0.50 \mathrm{M}_{\odot}$ which makes up $\lesssim 20-30$ per cent of all low-mass WDs (Brown et al. 2011). Combined with the fraction of low-mass WDs in the solar neighbourhood of $\sim 10$ per cent (Kepler et al. 2007), we estimate that $\lesssim 2-3$ per cent of all WDs are single low-mass WDs. These WDs cannot have formed through normal single star evolution as the Universe is simply not old enough.

Several explanations have been put forward to explain the existence of single low-mass WDs. A rather obvious possibility are merger events either during CE evolution or of two very low-mass WDs. However, when compared with observed samples these merger channels predict too few low-mass WDs and far too many single subdwarf B stars (Nelemans 2010; Brown et al. 2011).

As an alternative option it has been suggested almost two decades ago that close-in massive planets could lead to the formation of single low-mass WDs. In this scenario the stellar envelope of $\mathrm{a} \sim 1 \mathrm{M}_{\odot}$ star on the first giant branch is expelled in a CE like event but with the substellar companion either getting evaporated or merging with the giant (Nelemans \& Tauris 1998). While this is in general possible, rather massive planets or brown dwarfs are needed to unbind a substantial fraction of the envelope of the giant and it is not clear if massive close-in companions exist in large enough numbers to explain the fraction of low-mass WDs that seem to be single. 
As a third possibility strong mass loss or spontaneous ejection of the envelope close to the tip of the first giant branch for metal-rich stars has been proposed as an explanation for the existence of single low-mass WDs (Kilic, Stanek \& Pinsonneault 2007; Brown et al. 2011; Han, Podsiadlowski \& Eggleton 1994; Meng, Chen \& Han 2008). This scenario is consistent with the colour-magnitude diagram for the metal-rich cluster NGC 6791, which can be explained by a WD population dominated by low-mass WDs (Hansen 2005; Kalirai et al. 2007). However, a convincing alternative explanation for the colour-magnitude distribution of the WDs in this cluster was developed by Althaus et al. (2010) and García-Berro et al. (2011) who incorporated e.g. the energy released by the processes of ${ }^{22} \mathrm{Ne}$ sedimentation and constrained the number of single helium-core WDs in NGC 6791 to below 5 per cent. In addition, Miglio et al. (2012) measured mass loss on the giant branch in NGC 6791 and found values much too small to produce single low-mass WDs.

Finally, some single low-mass WDs might be the remnants of the companion stars to WDs that exploded as type Ia Supernovae. These supernovae evolved through the single-degenerate channel, either with red giant donors (Justham et al. 2009) whose envelopes have been stripped off by the explosion, or with close helium-star companions that might become low-mass WDs (Wang \& Han 2009). Indeed, recent observations of the hypervelocity helium-star US 708 , with a mass of $0.3 \mathrm{M}_{\odot}$, suggest that this is the most likely scenario for its formation (Geier et al. 2015). However, as it is not even clear to what extent type Ia Supernovae are produced by the single-degenerate channel, it remains elusive that we will soon be able to reliably estimate the formation rates of single low-mass WDs through these channels.

We here suggest a completely new formation mechanism for single low-mass WDs which is based on the new model for the evolution of cataclysmic variables (CVs) proposed by Schreiber, Zorotovic \& Wijnen (2016). CVs are semi-detached close compact binaries in which a WD accretes hydrogen-rich material from its low-mass MS companion star (e.g. Knigge, Baraffe \& Patterson 2011). Since the first binary population models for CVs have been published, a large number of CVs with low-mass WD primaries is predicted (e.g. de Kool 1992; Han, Podsiadlowski \& Eggleton 1995; Politano 1996) but not a single convincing candidate has been found observationally, which cannot be explained by selection effects (Zorotovic, Schreiber \& Gänsicke 2011; Wijnen, Zorotovic \& Schreiber 2015). We recently showed that the problem of the missing low-mass WDs in CVs can be solved if additional angular momentum loss generated by the mass transfer itself, so-called consequential angular momentum loss (CAML), causes especially CVs with lowmass WDs to merge (Schreiber, Zorotovic \& Wijnen 2016). This empirical approach represents an attractive possibility as other discrepancies between CV evolutionary models and observational population studies can be solved simultaneously (see also Nelemans et al. 2016). Amazingly, the new model may also solve a problem that is at first glance not related to CV evolution: The predicted large numbers of merging CVs should produce significant numbers of single low-mass WDs which might explain their existence. In other words, the CVs with low-mass WDs we seem to be unable to find might have merged and evolved into the single low-mass
WDs we have no convincing explanation for. In this letter we present binary population models that test this hypothesis and find that indeed merging CVs might be the dominant channel producing single low-mass WDs.

\section{BINARY POPULATION MODELS}

In order to predict the mass distribution of single WDs including those that formed through merging CVs, we performed population models of both single and binary stars. Assuming an initial binary fraction of 50 per cent, we generated an initial population of $10^{7}$ single stars and the same number of MS+MS binaries. We used the initialmass function of Kroupa, Tout \& Gilmore (1993) in the range $0.8-10 \mathrm{M}_{\odot}$ for the mass distributions of single stars and primary stars in binaries, and a constant star formation rate with an upper limit of 13.5 Gyrs for the age of the Galaxy (Pasquini et al. 2004). In the case of binaries, we assumed a flat initial mass-ratio distribution for secondary masses (Sana, Gosset \& Evans 2009), with a lower limit of $\mathrm{M}_{\mathrm{sec}}=0.069 \mathrm{M}_{\odot}$ (the brown dwarf limit in Knigge, Baraffe \& Patterson 2011). We also used a flat distribution in $\log a$ ranging from 3 to $10^{6} \mathrm{R}_{\odot}$ for the orbital separations (Popova, Tutukov \& Yungelson 1982), and no eccentricity. Solar metallicity was assumed for all stars.

The single-star evolution (SSE) and the binary-star evolution (BSE) codes from Hurley, Pols \& Tout (2000); Hurley, Tout \& Pols (2002) were used to evolve single and binary stars, respectively. The CE phase was modelled with an efficiency of $\alpha_{\mathrm{CE}}=0.25$ (Zorotovic et al. 2010) and with the binding energy parameter $\lambda$ properly computed assuming no contributions of recombination energy (see Section 2.2 in Zorotovic, Schreiber \& Parsons 2014). When a close WD+MS binary was formed, its subsequent evolution was calculated with our own code (Zorotovic et al. 2016). All zero-age WD+MS binaries were evolved to their current age assuming disrupted magnetic braking. CV evolution was calculated taking into account empirical CAML as suggested by Schreiber, Zorotovic \& Wijnen (2016). This revised model predicts that in a large number of systems with lowmass WDs unstable mass transfer is generated leading to a merger event instead of a CV with stable mass transfer.

The merging CVs should form single stars that then evolve into single WDs but this process is very complex. It is clear that following the merger event the systems enter a red giant configuration due to a rejuvenation of the hydrogenburning shell, i.e. the disrupted secondary forms a giant envelope around the WD (Shen, Idan \& Bildsten 2009). However, it is not clear if all the mass of the secondary is retained as an envelope or if, more likely, a fraction of this mass is expelled in a CE-like event. For the sake of simplicity, we decided to estimate the fraction of low-mass WDs produced by this channel considering two cases: $a$ ) the entire mass of the secondary star at the moment of merger is expelled, i.e. the mass of the WD remains unchanged; $b$ ) the entire mass of the secondary at the moment of merger is accumulated around the WD as a giant envelope. In case $b$ a giant with a total mass of $\mathrm{M}_{\mathrm{TOT}}=\mathrm{M}_{\mathrm{WD}}+\mathrm{M}_{\mathrm{sec}}$ and a core mass of $\mathrm{M}_{\mathrm{WD}}$ is generated. If $\mathrm{M}_{\mathrm{WD}}$ is larger than the final mass of a star with initial mass $\mathrm{M}_{\mathrm{TOT}}$, we assumed that it cannot grow more and therefore the final mass of the WD remains 

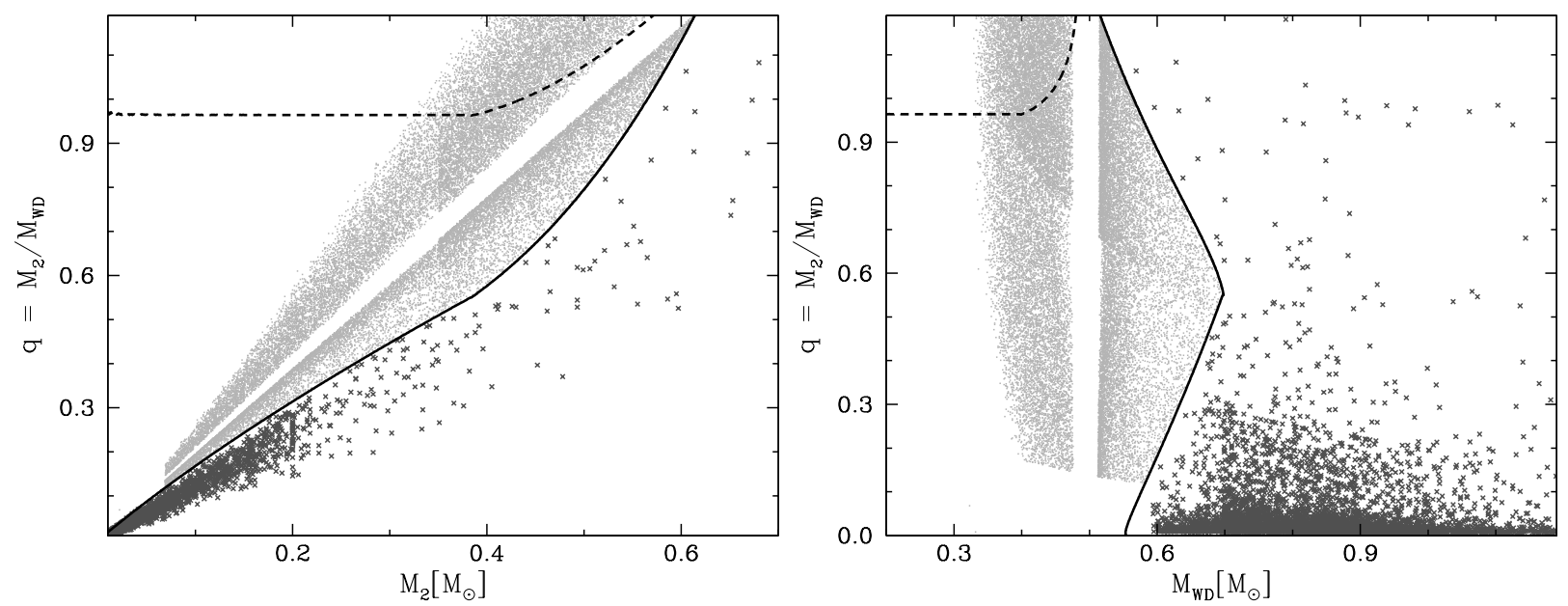

Figure 1. Mass-ratio versus secondary star mass (left) and WD mass (right) for CVs (black) and systems that merge (grey) due to unstable mass transfer. The solid line represents the critical mass-ratio for dynamically unstable mass transfer assuming empirical CAML as suggested by Schreiber, Zorotovic \& Wijnen (2016) while the dashed line is the critical mass-ratio obtained for classical CAML (King \& Kolb 1995). The new model predicts significantly more CV mergers than the classical model. Especially systems with low-mass WDs merge instead of becoming CVs.

Table 1. Number of single WDs produced by the different channels. "Others mergers" include mergers of MS+MS binaries and double WDs. The fraction of low-mass WDs with respect to the total number of single WDs is $\sim 1.6$ per cent and $\sim 0.6$ per cent for model $a$ and $b$ respectively.

\begin{tabular}{lrlrr}
\hline & \multicolumn{2}{c}{ All single WDs } & \multicolumn{2}{c}{$\mathrm{M}_{\mathrm{WD}} \leq 0.5 \mathrm{M}_{\odot}$} \\
channel & Number & Fraction & N. Case $a$ & N. Case $b$ \\
\hline \hline Single Stars & 4698675 & $71.4 \%$ & 0 & 0 \\
CE merger & 1413702 & $21.5 \%$ & 7544 & 7544 \\
CV mergers & 143532 & $2.2 \%$ & 93636 & 29554 \\
Other mergers & 325711 & $4.9 \%$ & 1635 & 1635 \\
\hline Total & 6581620 & & $102815(1.6 \%)$ & $38733(0.6 \%)$ \\
\hline
\end{tabular}

unchanged. If, on the other hand, the mass of the WD is smaller, we assumed that it can grow up to a mass given by the final (WD) mass of a single star with initial mass $\mathrm{M}_{\mathrm{TOT}}$. This is a simplified approach as the WD growth depends on the core-mass growth rate and the lifetime of the star on the giant branch, which are not necessarily equal to those of a star with initial mass $\mathrm{M}_{\text {Tот }}$. However, our simple approach is fully sufficient to get a reasonable estimate of the relative number of low-mass WDs formed by CV mergers. Detailed modelling of the merger process or the subsequent evolution of the resulting giant star are far beyond the scope of this paper.

In order to estimate the relative importance of CV mergers for the formation of single low-mass WDs, mergers of systems other than WD+MS that can produce single WDs were considered too. Merger events during the CE phase or in other close systems like MS+MS or double WD binaries were computed with BSE (see section 2.7 in Hurley, Tout \& Pols 2002 for details). MS+MS mergers contribute to the population of high-mass WDs, while mergers of double WDs or during the CE phase can form both lowmass and high-mass WDs.

\section{THE PREDICTED SINGLE WD MASS DISTRIBUTION}

The new model for CV evolution including empirical CAML predicts that in most $\mathrm{WD}+\mathrm{MS}$ binaries containing low-mass WDs dynamically unstable mass transfer is triggered after the MS star fills its Roche-lobe (Schreiber, Zorotovic \& Wijnen 2016). Therefore, significantly more mergers are predicted than by the previously assumed much weaker forms of CAML (e.g. King \& Kolb 1995). This is illustrated in Figure 1 which shows the mass ratio $q=\mathrm{M}_{\mathrm{sec}} / \mathrm{M}_{\mathrm{WD}}$ as a function of secondary star mass (left panel) and WD mass (right panel). The limits separating stable and dynamically unstable mass transfer are very different for the new empirical and the classical form of CAML (solid and dashed line in Figure 1, respectively). In the new model strong CAML for systems with low-mass WDs generates unstable mass transfer and the two stars merge.

The merging CVs generated by the new model enter a giant-like configuration and will become single WDs after the envelope is expelled. The exact number of low-mass WDs produced due to $\mathrm{CV}$ mergers depends on how much mass is lost during the merging process. We tested two prescriptions, i.e. we either assumed that all the mass of the secondary at the moment of merger is expelled (model $a$ ) or that 


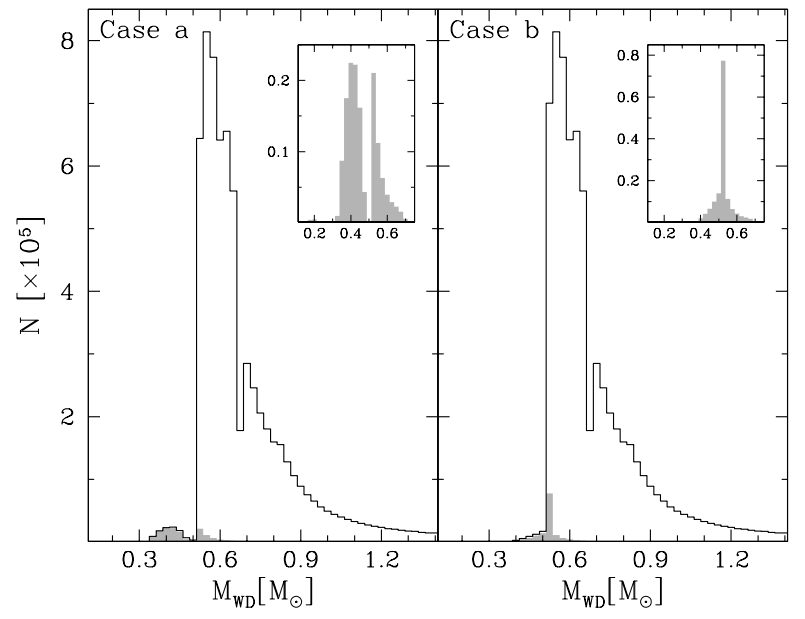

Figure 2. WD mass distribution of the single WDs predicted by our simulations. Left and right panels are for case $a$ and $b$ respectively (see text). Single WDs produced by CV mergers are shown in grey (a zoom is shown in the inset of each panel). The vast majority of single low-mass WDs $\left(\mathrm{M}_{\mathrm{WD}} \leq 0.5 \mathrm{M}_{\odot}\right)$ are formed by $\mathrm{CV}$ mergers.

all the mass is accumulated as an envelope around the WD (model $b$ ). The resulting mass distributions of single WDs for both models are shown in Figure 2. These distributions are of course dominated by single WDs descending from initially single stars, but significant numbers of low-mass WDs (with $\mathrm{M}_{\mathrm{WD}} \leq 0.5 \mathrm{M}_{\odot}$ ) are produced by CV mergers (represented by the grey histograms in Fig. 2, see also Table 1). Even if we assume that the whole mass of the secondary at the moment of merger is accumulated around the WD, the dominant channel producing single low-mass WDs in our simulation are merging CVs (Table 1 ).

Our simulations are based on several reasonable but uncertain assumptions that could in principal impact the predicted relative number of single low-mass WDs produced by CV mergers. Despite some rather convincing constraints (Sana, Gosset \& Evans 2009; Zorotovic et al. 2010), the most important uncertain parameters are probably the initial mass-ratio distribution and the CE efficiency. However, in Zorotovic et al. (2014) we determined the fraction of low-mass (helium-core) WDs among post common envelop binaries (PCEBs) for a wide range of CE efficiencies and two initial mass-ratio distributions and found only very weak dependencies. The fraction of low-mass WDs in PCEBs remained nearly unchanged in the range of $40-60$ per cent. This implies that also the fraction of low-mass WDs among CVs will not dramatically change if other assumptions for the mass-ratio distribution or the CE efficiency are used. Another uncertain parameter is the age of the Galaxy. However, assuming 10 Gyrs instead of 13.5 we found negligible changes in the resulting fractions of single low-mass WDs. Finally, the initial binary fraction of 50 per cent we assumed for all types of binaries independent of the primary star mass is probably incorrect. There is growing evidence that the binary fraction increases with increasing primary mass (e.g. Lada 2006). We thus may underestimate the number of descendants from more massive binaries which implies that we perhaps slightly underestimate the number of massive double WDs and maybe also the $\mathrm{CE}$ mergers of massive

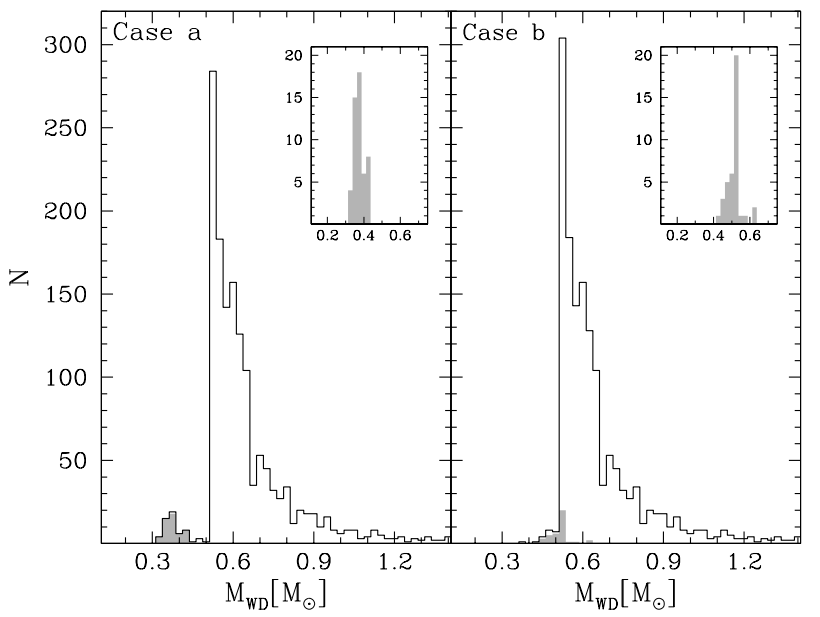

Figure 3. Same as in Figure 2 but after applying the filters in effective temperature and $g$ magnitude from Tremblay et al. (2016) described in the text.

stars. Both these channels can produce massive single WDs and we therefore might slightly overestimate the fraction of single low-mass WDs. However, the vast majority of binaries that form single WDs descend from systems with initial primary star masses in the range of $1-2 \mathrm{M}_{\odot}$, where the binary fraction is supposed to be similar to the $\sim 50$ per cent we assumed (e.g. Duquennoy \& Mayor 1991). Therefore, our simplified assumption does not affect the conclusions of this paper.

We conclude that if the empirical CAML model from Schreiber, Zorotovic \& Wijnen (2016) is included, the dominant channel producing single low-mass WDs might potentially be CV mergers. Our model predicts a fraction of lowmass WDs among single WDs of the order of one per cent. These findings are independent of model uncertainties.

\section{THE IMPACT OF SELECTION EFFECTS AND OBSERVATIONAL BIASES}

The fraction of single low-mass WDs predicted by the new empirical CAML model for CV evolution is of the order of one per cent. However, the WD mass distribution (shown in Fig. 2) represents the total population of single WDs predicted by our model and does not take into account observational biases and selection effects which need to be considered before our result can be compared with the observations.

Observational samples such as those from the Sloan Sloan Digital Sky Survey (SDSS, Abazajian et al. 2009) are magnitude-limited. To construct a magnitude-limited sample from our predicted sample of all single WDs we assigned a distance to each object in our final single WD sample using the same galactic distribution as Tremblay et al. (2016). According to these authors the spectroscopic completeness of DA WDs from SDSS data release 7 is constant over the parameter ranges $16000<\mathrm{T}_{\text {eff }}(K)<$ 22000 and $16.0<g<18.5$. In order to construct a subsample of WDs with these parameters we computed the effective temperature of the simulated WDs using the cooling tracks by Althaus \& Benvenuto (1997) for helium-core 
WDs (if $\mathrm{M}_{\mathrm{WD}} \leq 0.5 \mathrm{M}_{\odot}$ ) and Fontaine, Brassard \& Bergeron (2001) for carbon/oxygen-core WDs (if $\mathrm{M}_{\mathrm{WD}}>0.5 \mathrm{M}_{\odot}$ ) assuming only thick hydrogen envelopes (DA WDs). Using these temperatures and the WD masses, we calculated the absolute magnitude $M_{g}$ using the colour tables from Holberg \& Bergeron (2006, their pure hydrogen grids). The resulting WD mass distributions are shown in Figure 3. The fraction of single low-mass WDs is slightly increased $(3.9 \%$ in model $a$ and $1.3 \%$ in model $b$ ) and CV mergers remain the dominant channel producing low-mass WDs, with relative contributions nearly identical to the ones obtained before. These results do not change if we somewhat relax the rather strict selection criteria. For example, if we take into account WDs with reliable masses from spectral fitting, i.e. WDs with $\mathrm{T}_{\text {eff }}>12000 \mathrm{~K}$ (Kepler et al. 2007), and $i$ magnitudes covered by the SDSS Quasar survey (Richards et al. 2002 ), i.e. $15.5<i<19.1$, the fraction of single WDs with $\mathrm{M}_{\mathrm{WD}} \leq 0.5 \mathrm{M}_{\odot}$ is 3.3 per cent in model $a$ and 1.4 per cent in model $b$.

The slight increase of the fraction of low-mass WDs if observational selection effects and biases are taken into account is mainly caused by the longer MS lifetimes of the progenitors of low-mass WDs, which are relatively low-mass MS stars $\left(M_{\mathrm{i}} \lesssim 1.8 \mathrm{M}_{\odot}\right.$, see Figure 2 in Zorotovic \& Schreiber 2013). This implies that the evolutionary time-scale to form a CV with a low-mass WD is on average longer than to form a CV with a more massive WD. Therefore, the fraction of WDs too cool to be detected (e.g. by SDSS) is smaller for low-mass WDs because these are in general younger (and hotter) than more massive WDs. This effect is complemented by the fact that high-mass WDs cool faster than lowmass WDs. The final prediction of our model is thus that in observed samples $\sim 1-4$ per cent of all single WDs should be low-mass WDs mostly produced by CV mergers.

\section{CONCLUSION}

Despite some promising suggestions, the existence of single low-mass WDs has so far not been convincingly explained. In this paper we show that if consequential angular momentum loss as described by Schreiber, Zorotovic \& Wijnen (2016) is included in CV evolution, a significant number of single lowmass WDs is predicted to result from merging CVs. These single low-mass WDs should make up about $1-4$ per cent of all single WDs.

The only estimate of the fraction of single low-mass

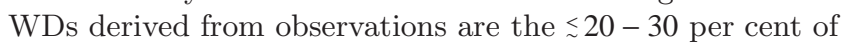
single WDs among low-mass WDs from Brown et al. (2011). If combined with the finding from Kepler et al. (2007) that low-mass WDs make up 10 per cent of all WDs in the solar neighbourhood, we find good agreement with the model predictions. The revised model for $\mathrm{CV}$ evolution suggested by Schreiber, Zorotovic \& Wijnen (2016) might therefore not only solve several long standing problems of CV evolution. By postulating that especially CVs with low-mass WDs merge, this model also offers an explanation for the existence of single low-mass WDs. It might well be that the low-mass WDs in CVs we expected to exist but never found, were hidden from us because they evolved into the single low-mass WDs whose existence we always struggled to explain.

\section{ACKNOWLEDGEMENTS}

We acknowledge financial support from FONDECYT (grants 3130559 and 1141269).

\section{References}

Abazajian, K. N., et al., 2009, ApJS, 182, 543

Althaus, L. G., Benvenuto, O. G., 1997, ApJ, 477, 313

Althaus, L. G., et al., 2010, ApJ, 719, 612

Brown, J. M., et al., 2011, ApJ, 730, 67

Brown, W. R., et al., 2010, ApJ, 723, 1072

de Kool, M., 1992, A\&A, 261, 188

Duquennoy, A., Mayor, M., 1991, A\&A, 248, 485

Fontaine, G., Brassard, P., Bergeron, P., 2001, PASP, 113, 409

García-Berro, et al., 2011, A\&A, 533, A31

Geier, S., et al., 2015, Science, 347, 1126

Han, Z., Podsiadlowski, P., Eggleton, P. P., 1994, MNRAS, 270, 121

Han, Z., Podsiadlowski, P., Eggleton, P. P., 1995, MNRAS, 272, 800

Hansen, B. M. S., 2005, ApJ, 635, 522

Holberg, J. B., Bergeron, P., 2006, AJ, 132, 1221

Hurley, J. R., Pols, O. R., Tout, C. A., 2000, MNRAS, 315, 543

Hurley, J. R., Tout, C. A., Pols, O. R., 2002, MNRAS, 329, 897

Justham, S., et al., 2009, A\&A, 493, 1081

Kalirai, J. S., et al., 2007, ApJ, 671, 748

Kepler, S. O., et al., 2007, MNRAS, 375, 1315

Kilic, M., Stanek, K. Z., Pinsonneault, M. H., 2007, ApJ, 671, 761

King, A. R., Kolb, U., 1995, ApJ, 439, 330

Knigge, C., Baraffe, I., Patterson, J., 2011, ApJS, 194, 28

Kroupa, P., Tout, C. A., Gilmore, G., 1993, MNRAS, 262, 545

Lada, C. J., 2006, ApJ, 640, L63

Marsh, T. R., Dhillon, V. S., Duck, S. R., 1995, MNRAS, 275, 828

Meng, X., Chen, X., Han, Z., 2008, A\&A, 487, 625

Miglio, A., et al., 2012, MNRAS, 419, 2077

Nelemans, G., 2010, Ap\&SS, 329, 25

Nelemans, G., Tauris, T. M., 1998, A\&A, 335, L85

Nelemans, G., et al., 2016, ApJ, 817, 69

Pasquini, L., et al., 2004, A\&A, 426, 651

Politano, M., 1996, ApJ, 465, 338

Popova, E. I., Tutukov, A. V., Yungelson, L. R., 1982, Ap\&SS, 88,55

Rebassa-Mansergas, A., et al., 2011, MNRAS, 413, 1121

Richards, G. T., et al., 2002, AJ, 123, 2945

Sana, H., Gosset, E., Evans, C. J., 2009, MNRAS, 400, 1479

Schreiber, M. R., Zorotovic, M., Wijnen, T. P. G., 2016, MNRAS, 455, L16

Shen, K. J., Idan, I., Bildsten, L., 2009, ApJ, 705, 693

Tremblay, P.-E., et al., 2016, MNRAS, 461, 2100

Wang, B., Han, Z., 2009, A\&A, 508, L27

Webbink, R. F., 2008, in Milone E. F., Leahy D. A., \& Hobill D. W., eds., Astrophysics and Space Science Library, Vol. 352, Springer, Berlin, p. 233

Wijnen, T. P. G., Zorotovic, M., Schreiber, M. R., 2015, A\&A, 577, A143

Zorotovic, M., Schreiber, M. R., 2013, A\&A, 549, A95

Zorotovic, M., Schreiber, M. R., Gänsicke, B. T., 2011, A\&A, 536, A42

Zorotovic, M., Schreiber, M. R., Parsons, S. G., 2014, A\&A, 568, L9

Zorotovic, M., et al., 2010, A\&A, 520, A86

Zorotovic, M., et al., 2014, A\&A, 568, A68

Zorotovic, M., et al., 2016, MNRAS, 457, 3867 


\section{L6 Zorotovic \&6 Schreiber}

This paper has been typeset from a $\mathrm{T}_{\mathrm{E}} \mathrm{X} / \mathrm{LAT}_{\mathrm{E}} \mathrm{X}$ file prepared by the author. 